
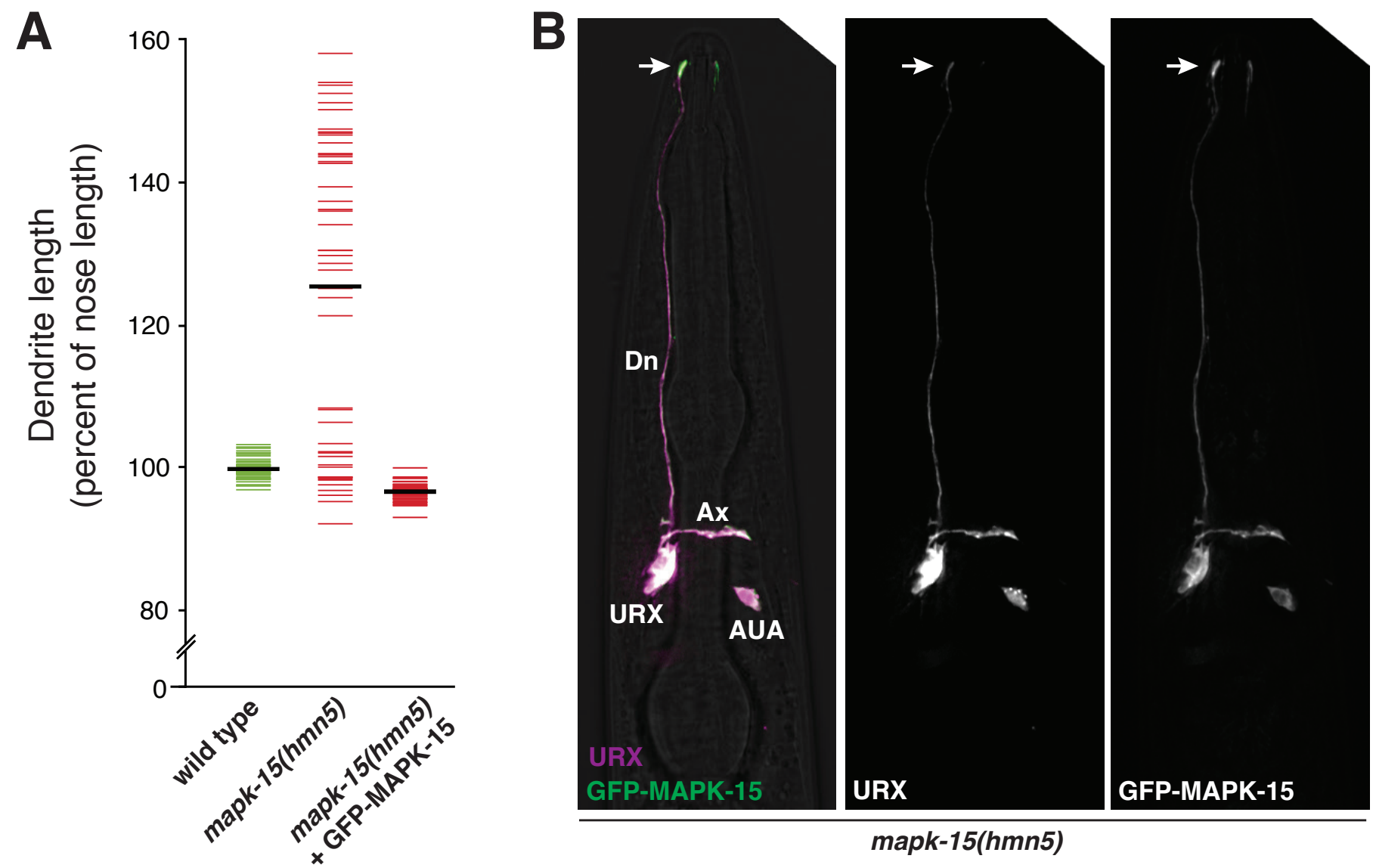

mapk-15(hmn5)

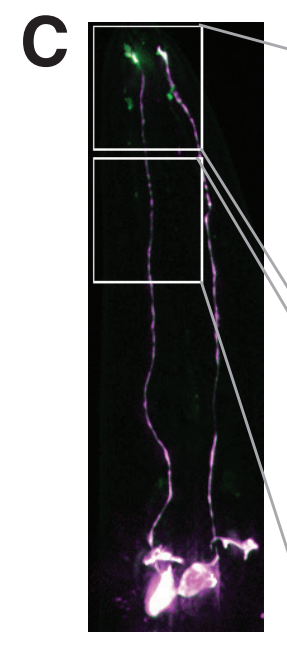

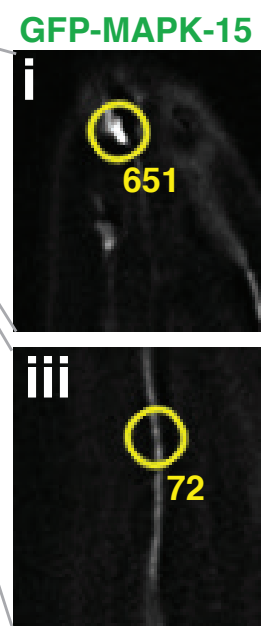

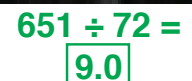

mCherry
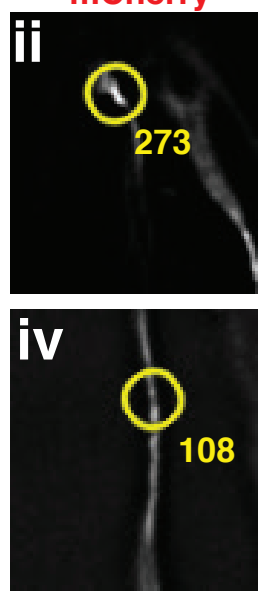

$273 \div 108=$

2.5

D

\title{
Relative enrichment
}
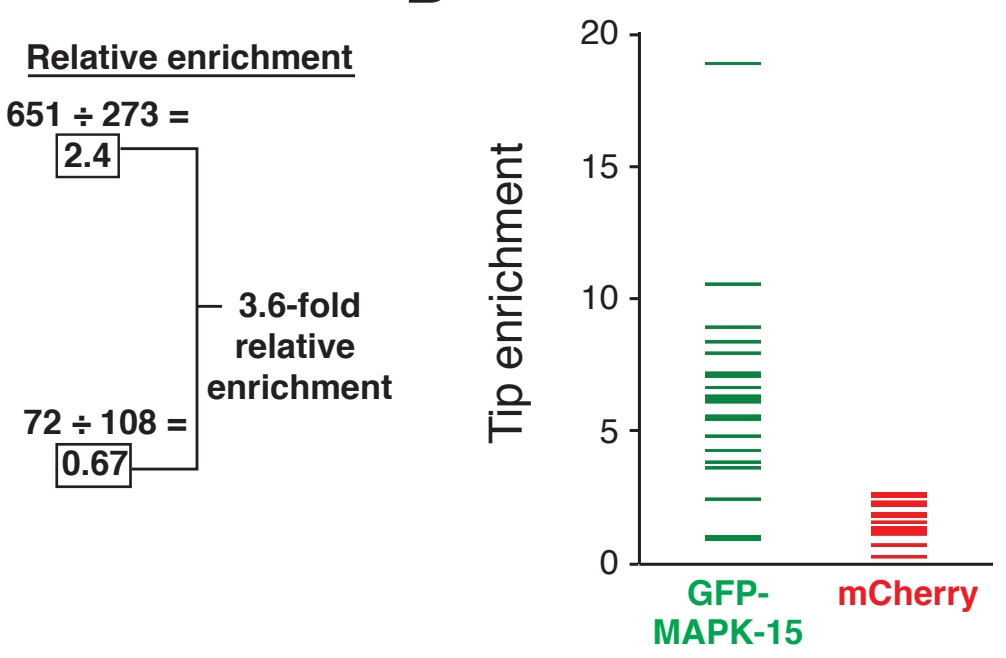

Tip enrichment

Supplemental Figure S3. A functional GFP-MAPK-15 is enriched at the dendrite ending

(A) Wild-type or mapk-15 animals with or without $f l p$-8pro:superfolderGFP-MAPK-15 were synchronized as two-day adults and dendrite and nose lengths were measured. Colored bars, individual animals; black bars, population averages. $\mathrm{n}=50$ in all cases. (B) mapk-15 mutant animal expressing flp-8pro:mCherry (URX) and 
flp-8pro:superfolderGFP-MAPK-15 (URX). Arrow, dendrite ending. (C) Schematic showing quantification of superfolderGFP-MAPK-15 enrichment at dendrite ending. Wild-type animals expressing flp-8pro:mCherry (URX) and flp-8pro:superfolderGFP-MAPK-15 (URX) were imaged. Fluorescence intensity of a 2- $\mu \mathrm{m}$ diameter circle (yellow) in a single optical plane at the dendrite ending (i, ii) or middle (iii, iv) was calculated and background fluorescence was subtracted to yield a corrected intensity (yellow numbers). Tip enrichment was calculated as the ratio of corrected intensities at the tip vs middle $(\mathrm{i} \div \mathrm{iii}, \mathrm{ii} \div \mathrm{iv})$. Relative enrichment of the GFP signal was calculated by first normalizing to mCherry $(\mathrm{i} \div \mathrm{ii}, \mathrm{iii} \div \mathrm{iv})$ to correct for local differences in cell volume, and then calculating the ratio of these normalized values $((\mathrm{i} \div \mathrm{ii}) \div(\mathrm{iii} \div \mathrm{iv}))$. (D) Tip enrichment of superfolderGFP-MAPK-15 and mCherry. Mean \pm SD: GFP, $6.3 \pm 3.9$, mCherry, $1.7 \pm 0.7 . n=20$. Colored bars, individual dendrites. 